\title{
Comprehensive Experimental Performance Analysis of DSR, AODV and DSDV Routing Protocol for Different Metrics Values with Predefined Constraints
}

\author{
Zafar Mehmood \\ Department of Computer Science and Information Technology, University of Gujrat, Gujrat, Pakistan \\ Email: zafar.mehmood@uog.edu.pk \\ Dr. Muddesar Iqbal \\ Department of Computer Science and Information Technology, University of Gujrat, Gujrat, Pakistan \\ Email: m.iqbal@uog.edu.pk \\ Xingheng Wang \\ School of Computing, University of the West of Scotland, Paisley Campus, Paisley, Scotland, UK \\ Email: xinheng.wang@uws.ac.uk
}

\begin{abstract}
A Mobile Adhoc Network is a multi-hop selfconfiguring network without any fixed infrastructure. Due to mobility of nodes, dynamic topology and highly dynamic environment, designing and implementing stable routing in Mobile Ad-hoc Networking is a major challenge and a critical issue. This paper analyses the performance analysis of on demand routing protocol, Dynamic Source Routing (DSR), Adhoc on Demand Distance Vector Routing (AODV) and table driven protocol, Destination-Sequenced Distance Vectoring (DSDV) using a network simulator NS2. Different types of test scenario have designed with fixed number of nodes but varying mobility. Different performance metric values like, throughput, delay, normalized network load, end to end delay, dropped packets, packets delivery ratio have been observed. The experimental results have been analysed and recommendation based on the obtained results has been proposed about the significance of each protocol in different scenarios and situations. The simulation results show that both protocols are good in performance in their own categories. We believe that findings of this paper will help the researcher to find the best protocol under predefined condition with varied mobility. We believe that this research will help the researcher to identify and further investigate any particular metrics value of AODV, DSR and DSDV.
\end{abstract}

Index Terms-Mobile Adhoc Networks, Ad Hoc On Demand Routing Vector (AODV), Dynamic Source Routing (DSR), Destination Sequenced Distance Vector (DSDV), network simulator NS-2.

\section{INTRODUCTION}

Mobile Adhoc Network (MANET) is a multi-hop selfconfiguring network without any fixed infrastructure of wireless devices (nodes, computer) connected by wireless links. Mobile Adhoc networking is broadly categorized into two types: Reactive Routing Protocols and Proactive Routing Protocols. AODV (Adhoc on
Demand Distance Vector) [1], DSR (Dynamic Source Routing) [2] and DSDV (Destination-Sequenced Distance Vectoring) [3] are major mobile Adhoc network (MANET) routing protocols that has been investigated and researched for many years. MANET does not require centralized command and control. Thus the network is suitable for applications requiring rapid deployment. The most prominent applications of mobile Adhoc networking protocol is military communication network in: battle fields, different types of rescue operation in emergency, under sea operations, monitoring different environmental changes and effects and space study. Because of its "in-the-air" arrangement quality and reasonably minimal cost of installation, Mobile Adhoc Networks are also used in different applications due to its reasonable installation price than its infrastructure counterparts. [4]

In past many researchers have performed the experimental analysis of table driven protocol and on demand routing protocol by analyzing their throughput, packet delivery ration, end to end delay and normalized routing load based on different predefined constrains. In paper [5], table driven protocol DSDV, and On Demand Routing protocol AODV and DSR has been analyzed with the fixed mobility and increasing number of nodes to compare throughput, delay, normalized routing load, number of sent packets. The result shows that in less dense environment DSR shows better performance than AODV and DSDV, but with increasing number of nodes AODV slightly changed his behavior and shows better result than DSR and DSDV by measuring throughputs, packet delivery ration, number of sent and received packets, but end to end delay is still better in DSR even in more dense situation. Paper [6] shows performance investigation of DSDV, AODV and DSR under 
predefined constraints to evaluate end to end delay, packet delivery ratio and throughput. The result and graph shows that DSDV and DSR shows better performance in term of throughput as compared to AODV in less dense situation, but when the number of nodes increases, AODV protocol change drastically and show its suitability for large environment.

In paper [7] table driven protocol DSDV, source base routing, DSR and table base routing AODV are compared under predefined constrains like 100 nodes, with $10 \mathrm{~m} / \mathrm{s}$ mobility, varying pause time between 0 to 50 seconds and different terrain area of $100 * 100,1000$ * 1000 meters. Simulation result shows that DSR shows more optimal behavior in terms of throughput in terrain area of $100 * 100$ meter than $1000 * 1000 \mathrm{~m}$. Result for AODV shows the same optimal behavior in small terrain area under the same condition, but comparison for both AODV and DSR for $100 * 100$ meter and $1000 * 1000$ meter terrain area with same condition, DSR Routing Protocol shows better performance for $100 * 100$ meter terrain area than $1000 * 1000$ meter terrain area for DSR and $100 * 100$ meter terrain area and $1000 * 1000$ meter terrain area for AODV.

Paper [8] highlights performance analysis for the metrics values numbers of packets receive vs. nodes, throughput vs. nodes and packets dropped vs. nodes for different routing protocols, DSR, AODV and DSDV. This paper shows that Received packets for DSR are more significant in number than AODV and DSDV with varying numbers of nodes and fixed simulation time. DSR and OLSR shows much better transfer time than AODV and DSDV. Analysing throughput vs. node DSR shows slightly better performance than AODV and DSDV, and AODV shows better result in terms of packets receiving than DSDV. Analysis of packets dropped vs. nodes reflects that in DSR less number of packets is dropped due to its source based routing nature than AODV and DSDV.

The main objective of this research paper is to compare and analyse the performance of DSDV, AODV and DSR, [9], by comparing metrics values like throughput, packet delivery ratio, number of sent and received packets, end to end delay, normalized routing load, number of dropped data packets and number of dropped bytes under predefined constraints, like simulation area, fixed number of nodes, constant pause time, 900 seconds simulation time, 512 bytes packets size, 15 number of connections, maximum packets size is 50 with varying mobility of nodes from $5 \mathrm{~m} / \mathrm{s}$ to $35 \mathrm{~m} / \mathrm{s}$. The novelty of this paper is to provide a complete and thorough comparison of AODV, DSR and DSDV for their different performance parameters under predefined constraints, while in past research papers either the two or three routing protocols are compared for their few performance parameters which just highlight which protocol is best in terms of throughput, normalized routing load, delay and packet delivery ratio under defined constraints. In this paper we include 8 different performance metric values on the basis of these different matric values we can conclude that which protocol is best for a particular metric value under particular scenarios at one place

The remainder of paper is organized in the following sections. Section 2 covers working mechanism of reactive and proactive routing protocols. Different mobile Adhoc routing protocol are discussed in section 3 Section 4 highlight the Proactive Protocol, DSDV, and Reactive Protocol, AODV and DSR in detail, in section 5 we described the methodology, which shows simulation tool, in our case that will be NS2 environment, simulation parameter and Metrics values used in our work simulation, Section 6 demonstrate the result and analysis, section 7 present conclusion, section 7 contained references.

\section{ROUTING PROTOCOLS FOR ADHOC NETWORKS}

In mobile Adhoc networking, routing means exchange/ transfer of information or statistics from one node to another; the node may be a device or a computer. The main objective of routing is to find and maintain routes between source and intended destination in a highly dynamic environment where network topology varies more frequently. Mobile Adhoc routing protocols are classified into two sub types, unicast routing and multicast Adhoc routing protocols. In Unicast routing protocol, forwarding means that one source node transmit data and control packets to a single destination node. In Multicast routing protocols a source node may wish to transit the same data or control packet to more than on destination. Unicast routing protocol are further sub divided into proactive, reactive and hybrid routing protocols [5] [9].

\subsection{Proactive Routing Protocols}

The Proactive Routing Protocols maintain the rout information proactively. Every node maintained information about network and frequent topological structure in their respective routing table. A periodic update message is required to keep the routing table consistent and updated after every significant change in the network structure and nodes positions. Among many proactive routing protocols, DSDV is one of the most prominent table driven routing protocol.

\subsubsection{Destination-Sequenced Distance Vectoring}

DSDV is one of the earliest prominent routing MANET protocols proposed for mobile Adhoc networking. In DSDV a routing table is sustained at every station/ node in the network having shortest path information to the entire neighbour's node and the first node on the shortest path to every other node. Within the network data and control packets are transmitted from one node/station to other node/station by using routing table which store at each node.

Every station/ node in the network preserves record of all neighbouring routes. Since all tables driven routing protocol are proactive in nature, their routing table must be updated. To maintain the consistency of routing table each station/node of the network exchanges its routing table with all its neighbour nodes from time to time after 
particular intervals or any significant topological change observed in the network [5] [10]. A random sequence number is maintained by each entry in the routing table originated by destination node, if this sequence number is even it means link is alive, and if link is broken sequence number is set to be odd. Destination node set the sequence number and then exchanged with the source node. The major goal of proactive routing protocol is to find path with least hop count to the destination [11].

Due to proactive nature, DSDV requires continues update of routing table, so if there is no communication taking place between the nodes, it still requires battery power and bandwidth. That's why it is not suitable for highly dynamic networks [12].

\subsection{Reactive Routing Protocols}

Reactive Routing Protocol is also called "On-Demand Routing Protocol", reactive routing protocol establishes a path when actually it desires to communicate with another node in the network. Reactive routing protocol uses the techniques of distance vector routing algorithm, where vectors are used to store and maintain information about the cost (number of hop count and other resources) and route to the intended destination node. AODV and DSR are the most prominent routing protocols of reactive routing protocols.

\subsubsection{Dynamic Source Routing}

Reactive routing protocols, as compared to proactive routing protocols are entirely on-demand routing protocol, on- demand routing protocol, DSR is mainly functioned using route discovery and route maintenance. In DSR, when two nodes want to communicate with the intended destination node, it first checks its rout table for any available route to the destination, if it is available and valid it starts sending the data packets, and if there is no available route in the source node route cache, the source node initiates process for route discovery (RREQ) to find a route to the target destination node. The source node initiates and locally broadcasts a route request (RREQ) packet, having the following header: the intended destination and a unique identifier/sequence number from the source node. Each intermediate node after receiving the RREQ performs three actions, 1 . Is it an intended destination node by comparing the destination IP address with its own, if yes generate route reply (RREP), 2. By comparing he sequence number, the intermediate node decides whether it has recently seen this request? If yes simply discard the RREQ packet, 3. if not, then simply the intermediate node appends its information to the packet header and rebroadcast the RREQ. The same process will be continue until the RREQ reaches to the destination node, when the RREQ arrives at destination a reverse process RREP (route reply) is started back to the source node. RREP packet includes a copy of accumulated list of address of the all the intermediate nodes from the RREQ packet. After receiving the source node, the source node caches the new route from the RREP packet and store in its route cache for future use and to transmit the data packets to its target destination. [11] [13] [14] [15]
Route maintenance process is taking place if the source node facing some problem in transmitting the data packets along a specified path to the target, if that link/ path has damaged. For example if the source node want to communicate with the target destination, due to the mobility of nodes and topological changes in the network topology, the two nodes may move too far apart, leave or join the network. Since DSR is source routing, during the packet transmutation/sending process, the source node lists in the packet header the complete sequence/order of node through which the packet has to be travelled, each intermediate node on the route forward the packet to its neighbours listed in the packet header, and wait for the conformation, conformation can be carried out by different methods, like a network layer acknowledgment, passive acknowledgment, or link-layer acknowledgment, after transmission if the sending node does not receive any conformation, it retransmit the packet to the next node for a limited number of time, and if still the node is not acknowledged, the node generate route error (RERR) to the initiator/ source node of the packet, which is a signal of link failure from itself to the next node. The source node than updated its route table and route cache by removing this link and broadcasting to the entire network that the specific link is no more valid for transmission, the source node than check its route cache for another route to the destination, if available it start using this route, otherwise it generate a new RREQ to find a path to the indented destination. [5] [11]

\subsubsection{Adhoc On Demand Distance Vector}

Reactive routing protocol, AODV is completely ondemand routing protocol like DSR which forms a route on demand when a sending node/station desire a route to the destination. The main difference between AODV and DSR is their source routing features. In DSR everything is included in the packet header which is necessary for transmission. AODV is derived from both DSR and DSDV; AODV use RREQ properties and route maintenance procedures from DSR and sequence number, hop by count, and periodic update from DSDV routing protocol. AODV operates on the functionality of distance vector technology. Opposite to the DSR, in AODV each node/station maintains a routing table which contains routing information to all the nodes in the network. When a source node/initiator want to communicate with another node in the network, first the source node checks its routing table. If there is no route available for the intended destination, it will broadcast, but that broadcast will be an IP limited broadcast. Every node in the limit of hop count of source node will receive the RREQ message and will create the route back to the source node through the path they get from the RREQ [16]. The route request packet header contains the following information: Source node IP address, broadcast id, current sequence number, and the most current sequence number for the destination. The intermediate receiver node can take one of the following actions, if it is the intended target node, it initiates route reply process (RREP) to the source node with matching sequence number greater or equal to that contained in the RREQ packet header, if not then the 
intermediate node will rebroadcast to next hop count limit, all the nodes/stations in the broadcast range retain trajectory of the RREQ's source IP address and broadcast id. After some time if the same node receives RREQ, it will be discarded if it has been processed earlier. If the source node receives a RREP message from another node, if this route reply packet is holding a greater sequence number or same sequence number as it records from the earlier RREP, it may bring up to date its directionfinding information and record the latest better path for future transmission [11]. When a RREP is sent to the source node, all the nodes along the path keep records of this route to the destination through this packet. The source node will use any active link for transmission of data to the destination, a link will be considered active if it is used for transmitting data or controlled traffic by a node to any other node beside the path. In case of link failure in the active route, the upstream mobile node generates a RRER message to the source node, that this link is no more available for transmission. The source node then re-initiate the route discovery. Since in AODV a node may receive multiple RREQ and RREP, there may be a chance of looping, to avoid routing loop a fresh route will be used for transmission, for that purpose a destination sequence number will be used. Sequence number will only by changed by the host in monotonically growing mode. A route will be considered fresh enough if it contains a larger sequence number or equal sequence number with minimum hop count, both RREQ and RREP will carry the sequence number. To avoid the stale/old path the RREP packets will contains larger or equal sequence number to the one that are listed in corresponding RREQ packet. If there are several paths with the same sequence number the shortest path will be used for transmission [5] [17] [18].

\section{Methodology}

To perform our required performance analysis we use a network simulation tool, NS-2, list of parameters under which performance can be analysed, metrics value, which will be analysed and simulation set up.

\subsection{Simulation Tools}

Designing and constructing a model of real system and conducting experiments on this model to analyse the behaviour of system or to evaluate the system for different strategies is called simulation, to analyse the behaviour of different routing protocol of wired and wireless network different simulator are being used by the researcher according to their requirements, like NS-2, NS-3, OPNET and OMNet ++. Here in this research paper we use NS-2 network simulator. To study the dynamic nature of wireless communication we need event driven simulator. NS2 is a very good event driven simulation tools that can be used to simulate wired and wireless network functions and protocols. Different $\mathrm{C}++$ classes implemented in NS-2 are using for creation different network components like number of nodes in the network, different types of queues, link between the nodes etc. all the network components are created, plugged and configured in NS-2 from TCL [19] [20].

\subsection{Metrics Value}

To evaluate the performance of proactive routing protocol, DSDV and reactive routing protocols, AODV and DSR, we evaluate the following metrics values.

Throughput: Throughput is measured as, average of successful message delivery over a network. It is measured as the ratio of the amount of received data to the total simulation time.

Delay (end to end delay): Delay is the time taken by a bit of data to move across the network. The average end to end delay is calculated by taking the sum of all packets sent and received divided by total number of packets.

Packet delivery ratio: Packet delivery ratio is the ratio of packets generated by CBR resources and the number of delivered data packets to the destination.

Normalized routing load: Ratio of the total number of routing packets transmitted from the source node to data packets delivered at destination. Each hop wise transmission of routing packets is calculated one transmission.

Number of dropped packets: A packet is buffered if the destination is not known when it is arrived in a network layer otherwise it is forwarded to the destination. A packet is lost when buffered becomes full or time limit to store the packet in the buffer exceeds.

\subsection{Simulation Setup}

In this paper we tested the packet delivery ratio, throughput, normalized routing load, average end to end delay, no of dropped packets, no of dropped bytes, no of sent packets and the number of received packets using AODV, DSR and DSDV routing protocol with selfcreated scenarios where the total number of nodes are 50 uses 15 connections, pause time is 50, traffic type CBR, maximum packet size used in simulation is 512 Bytes, and the mobility varied starting from $5 \mathrm{~m} / \mathrm{s}, 10 \mathrm{~m} / \mathrm{s}, 15$ $\mathrm{m} / \mathrm{s}, 20 \mathrm{~m} / \mathrm{s}, 25 \mathrm{~m} / \mathrm{s}, 30 \mathrm{~m} / \mathrm{s}, 35 \mathrm{~m} / \mathrm{s}$ in $1000 * 1000$ meter terrain area. Two ray ground propagation is used in wireless channel with Omni antenna and 802-11 Mac Type. The simulation is taken to be of 900 seconds.

\begin{tabular}{|ll|}
\hline Number of Nodes & 50 \\
\hline Pause Time & $50 \mathrm{sec}$ \\
\hline Simulation Time & $900 \mathrm{sec}$ \\
\hline Mobility & $5,10,15,20,25,30,35 \mathrm{~m} / \mathrm{s}$ \\
\hline Traffic Type & CBR \\
\hline Packet Size & 512 Bytes \\
\hline Scenario Size & $1000 * 1000 \mathrm{~m}$ \\
\hline Channel Type & Wireless Channel \\
\hline RadioPropagation Model & Two Ray Ground \\
\hline Mac Type & $802-11$ \\
\hline Link Layer Type & LL \\
\hline Maximum Packets & 50 \\
\hline Protocols & AODV,DSDV,DSR \\
\hline Number of Connections & 15 \\
\hline Antenna model & Omni Antenna \\
\hline
\end{tabular}

Fig. 1. Simulation parameter 


\section{Simulation Result}

Here in this section we compare and analyse the result of all the three protocols comprehensively, by comparing the following metrics values, ratio of packet delivery friction, end-to-end delay, the number of sent packets, number of received packets, normalized routing load, number of dropped data packets, number of dropped data bytes and throughput We display all the results in the form of graphs. In the following section we discuss each graph result to highlight the performance of AODV, DSR and DSDV.

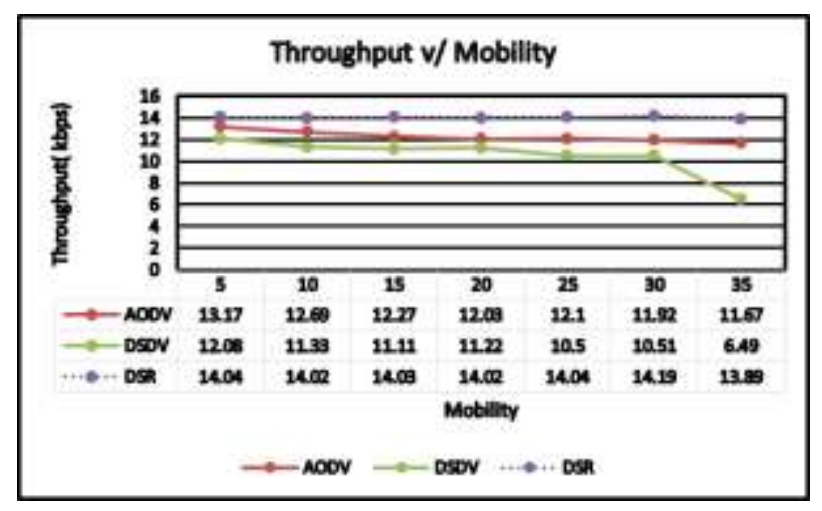

Fig. 2. Mobility versus throughput

Under the following constraints of performance parameter, when number of nodes are 50 , mobility varies from 5 to $35 \mathrm{~m} / \mathrm{s}$, pause time $50 \mathrm{sec}$, packet size 512 bytes and simulation time is $900 \mathrm{sec}$, The Fig. 2 highlights that DSR have most consistent throughput than AODV and DSDV due to its source base routing, whereas AODV show better performance than DSDV. With the increasing mobility throughput of DSDV is greatly reduces because of more flooding.

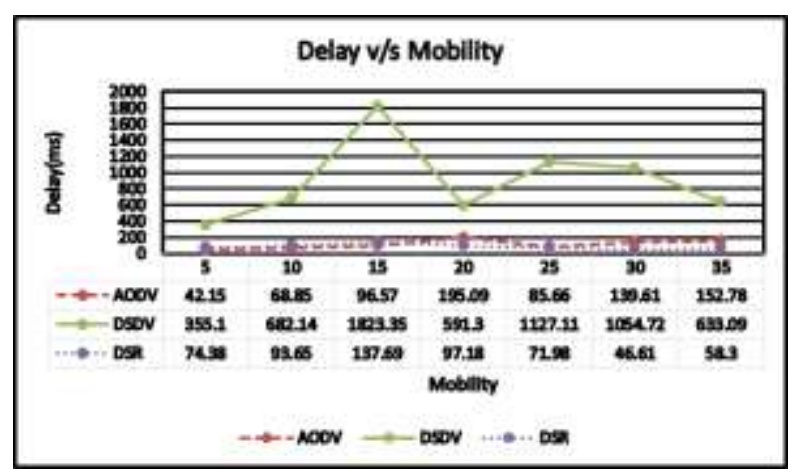

Fig. 3. Delay v/s Mobility

Under the following constraints of performance parameter, when number of nodes are 50 , mobility varies from 5 to $35 \mathrm{~m} / \mathrm{s}$, pause time $50 \mathrm{sec}$, packet size 512 bytes and simulation time is $900 \mathrm{sec}$, it is concluded in Fig. 3 that DSDV have more Delay than DSR and AODV, because of its proactive nature DSDV requires to establish routes between nodes prior to communication. In AODV and DSR, DSR shows better performance than AODV in delay property due to its aggressive use of route cache.

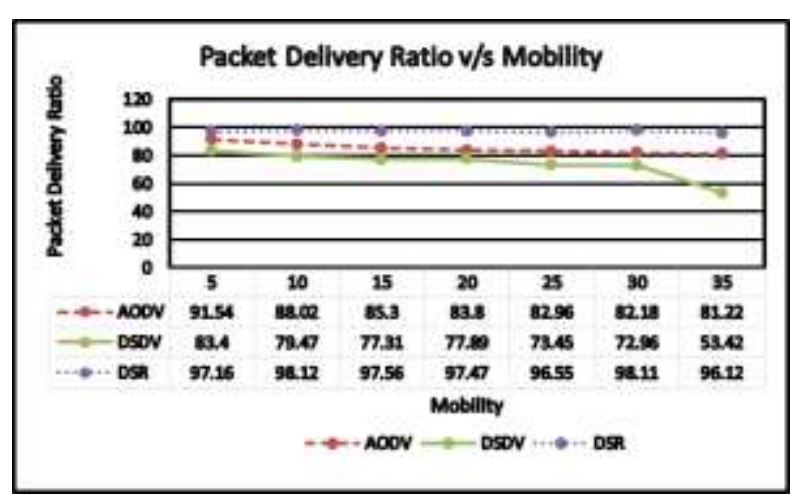

Fig. 4. Packet delivery frictions vs. Mobility

Fig. 4 highlights simulation results for packet delivery ratio using NS-2 simulator. When the number of nodes are 50 , mobility varies from 5 to $35 \mathrm{~m} / \mathrm{s}$, pause time 50 sec, packet size 512 bytes and the simulation time 900 sec.

Fig. 4 shows that DSR is more stable and consistent in packets delivery. Throughput and packet delivery ratio are correlated to each other. Since DSR is source base routing and using route cache mechanism due to which multiple routes are available in case of link failure, where AODV maintains only on entry for a route in its route table. With the changing mobility there is no change in packet delivery ratio for DSR, while comparing AODV and DSDV result shows that AODV is more prominent than DSDV.

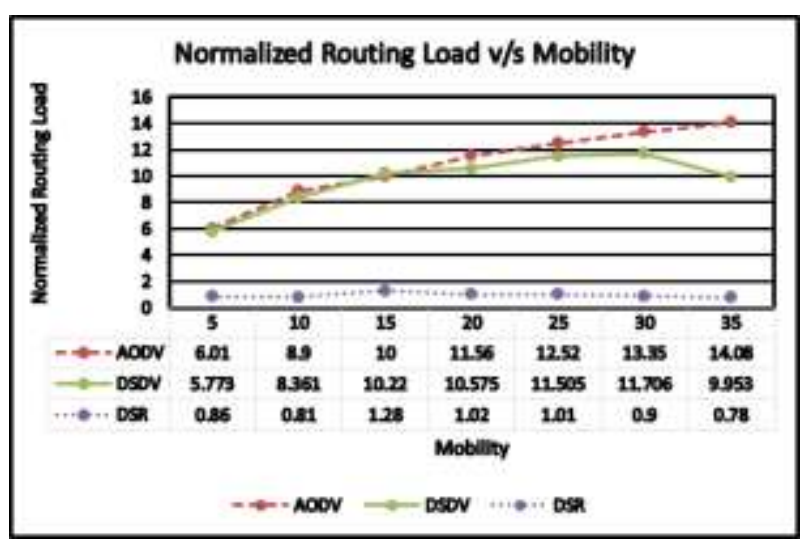

Fig. 5. Normalized routing load v/s Mobility

Under the following constraints of performance parameter, when number of nodes are 50 , mobility varies from 5 to $35 \mathrm{~m} / \mathrm{s}$, pause time $50 \mathrm{sec}$, packet size 512 bytes and simulation time is $900 \mathrm{sec}$, it is concluded from the Fig. 5 that normalized routing load is minimum in DSR as compared to AODV and DSDV, whereas DSDV shows better result for NRL then AODV.

Under the following constraints of performance parameter, when number of nodes are 50, mobility varies from 5 to $35 \mathrm{~m} / \mathrm{s}$, pause time $50 \mathrm{sec}$, packet size 512 bytes and simulation time is $900 \mathrm{sec}$, a conclusion can be drawn from Fig. 6 that No of dropped packet (bytes) ration is very less in DSR then AODV and DSDV, whereas DSDV dropped more packets (bytes) than AODV, because in both the protocols with greater 
mobility all the nodes in the network may leave or join the network, by losing discovered path and excessive hello messages, where as in AODV the destination replies only once to the request arriving first and ignores the rest and the routing table maintains at most one entry per destination.

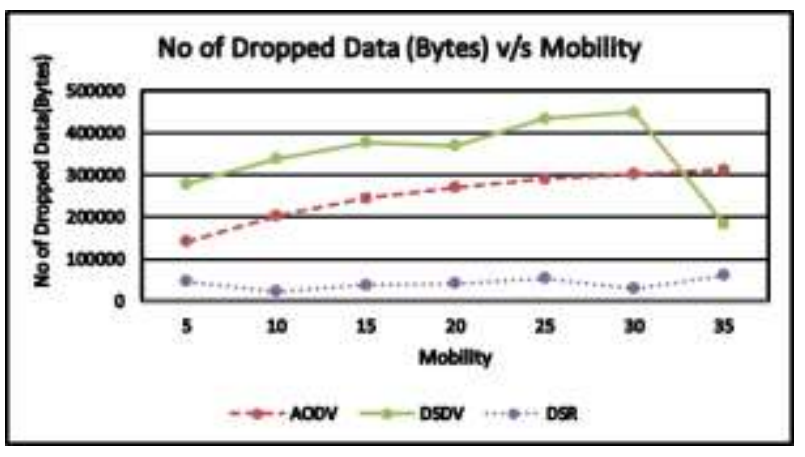

Fig. 6. No. of dropped data (bytes) v/s Mobility

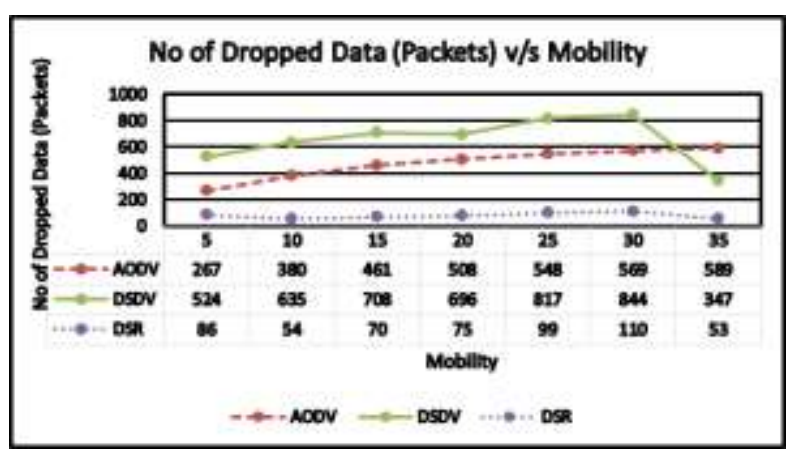

Fig 7. Number of dropped packets v/s Mobility

Under the following constraints of performance parameter, when number of nodes are 50, mobility varies from 5 to $35 \mathrm{~m} / \mathrm{s}$, pause time $50 \mathrm{sec}$, packet size 512 bytes and simulation time is $900 \mathrm{sec}$, it is concluded from the Fig. 7 that DSR shows better performance than AODV and DSDV, whereas DSDV show poor performance then AODV. But when mobility getting increases from 30 to $35 \mathrm{~m} / \mathrm{s}$, DSDV behaviour drastically changes and shows better performance than AODV.

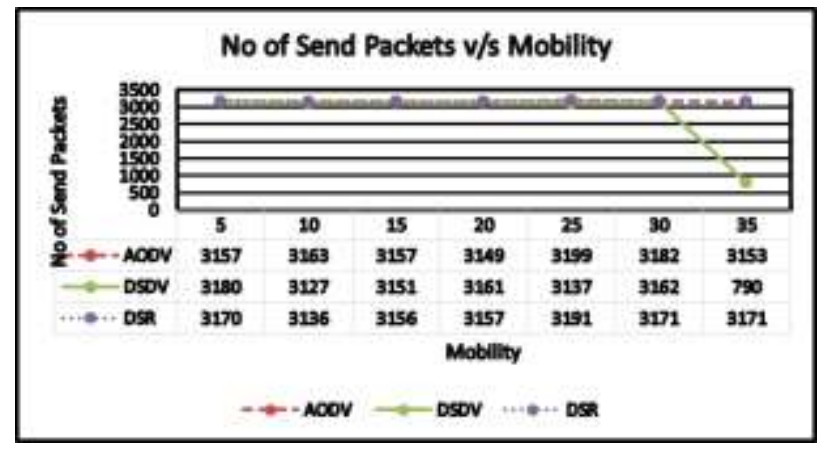

Fig. 8. No of sent packets v/s Mobility

Under the same constrained defined in Fig.7, Fig. 8 give the following results, Average sending packets rate of DSR is slightly better than AODV and DSDV routing protocols, because DSR using route cache mechanism which improve the route discovery process, and AODV inherits route discovery process from DSR, which causes significant improvement in sending packets. DSDV sending rate suddenly decrease at highest mobility level due to table driven approach.

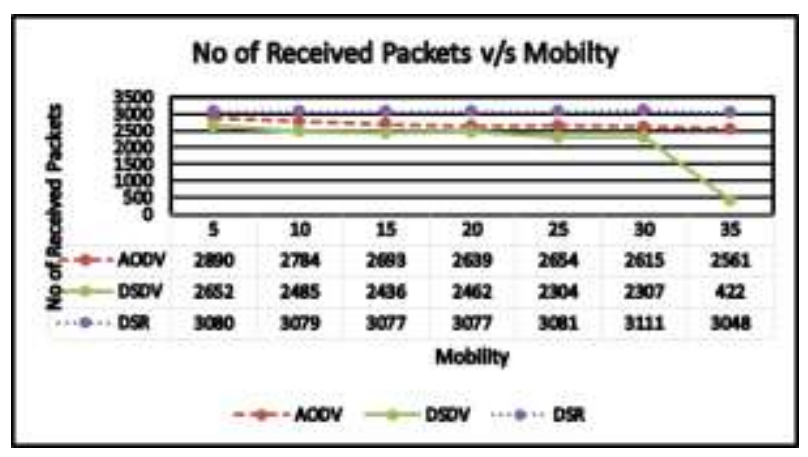

Fig. 9. No of received packets v/s Mobility

Under the same constrained defined in previous figures Fig. 9 highlights that amount of packets received by destination varies for all routing protocols, with varying mobility. The values of received data packets values are more stable for DSR as compared to AODV and DSDV. As mobility increases amount of received packets in AODV protocol increases but in the case of the DSDV when mobility reaches $30 \mathrm{~m} / \mathrm{s}$ it shows very poor performance by receiving packets.

\section{CONCLUSION}

After plotting graph and tables now we are able to discuss, conclude and analyse the performance of table driven routing protocol, DSDV and on demand routing protocol, AODV and DSR. We analyse the following metrics values, packet delivery ratio, throughput, normalized routing load, average end to end delay, no of dropped packets, no of dropped bytes, no of sent packets and the number of received packets are evaluated according to varied mobility. Different self-created scenario files are used in the simulation; each scenario with different mobility time e.g. with $5 \mathrm{~m} / \mathrm{s}, 10 \mathrm{~m} / \mathrm{s}$ up to $35 \mathrm{~m} / \mathrm{s}$ mobility Our study provides the most favourable result because it is fully based on simulation and analysis. With the help of tables and graphs every case explains the evaluation of parameter of three protocols AODV, DSDV and DSR. We draw graph and table for each metrics value, packet delivery ratio, throughput, normalized routing load, average end to end delay, no of dropped packets, no of dropped bytes, no of sent packets and the number of received packets for all the three protocols. Every protocol has its advantages and disadvantages. Our graphs and tables show that on demand routing protocol, AODV and DSR performed well and provide better results as compared to table driven protocol, DSDV. While comparing AODV and DSR, result shows that, DSR outclass AODV in number of received packets, number of dropped packets, number of dropped bytes, normalized routing load, DSR is more consistent and shows better packet delivery ratio than 
AODV, in throughput there is a slight change showing again that DSR is better than AODV. The only metrics value in which AODV out class DSR is average end to end delay. These entire conclusions are made, when the numbers of node are fixed, but surely AODV will show better performance in denser environment than DSR. It is further concluded that each Routing protocol has its own significance under specific circumstances and constraints. The tables and graphs in this paper only show performance analysis based on only one scenario, "under varied mobility" and if we change the scenario i.e. increase the number of nodes surely all the three protocols will show slightly different behaviour.

\section{REFERENCES}

[1]. C. Perkins, E. Belding-Royer, and S. Das, "Adhoc ondemand Distance vector (AODV) Routing", Proceedings of the $2^{\text {nd }}$ IEEE Workshop on Mobile Computing Systems and Applications (WMCSA) (pp.90-100), July 2003.

[2]. D. B. Johnson, D. A. Maltz, and Y. Hu, "The dynamic source Routing protocol for mobile Adhoc networks (DSR)", April 2003.IETF Internet Draft, draft- ietfmanet-dsr-09.txt,(work in progress).

[3]. Hemanth Nerra, Yufei Cheng, Egemen K, Cetenakia, Justin R. Rohar, "DSDV routing protocol implementation in ns3", SIMUTools '11 Proceedings of the 4th International ICST Conference on Simulation Tools and Techniques, pp. 201 - 205, March 22 - 24, 2012

[4]. Asis Nasipuri, "Mobile AdHoc Networks", NC 282230001

[5]. S. Gupta, C. Kumar, S Rani and B. Bhushan, "Performance comparison of routing protocols using different mobility models", IJMECS, vol. 4, no. 8, pp.5461, 2012.

[6]. S. D. Khatawkar, K. K. Pandyaji, R. M. Patil, V. M. Sali, U. L. Kulkarni, "Performance Comparison of DSDV, AODV, DSR, Routing protocols for MANET", International Conference on Computer Networks and Communication Systems (CNCS 2012) IPCSIT vol.35(2012) () (2012) IACSIT Press, Singapore

[7]. B. Bhushan, S. Gupta, C.K.Nagpal, "comparison of on Demand Routing Protocol", International Journal of Information Technology and Computer Science(IJITCS), PP.61-68, DOI: 10.5815/ijitcs, 2013.

[8]. Madhav sharma, Professor Rajeshwar Lal Dua, "COMPARISON OF DIFFERENT ROUTING PROTOCOLS (DSR \& AODV) ON BEHALF OF EVALUATION OF DIFFERENT ROUTING PARAMETERS WITH CONSTRAINTS ", IRACST International Journal of Computer Networks and Wireless Communications (IJCNWC), ISSN: 2250-3501 Vol.2, No.3, June 2012.

[9]. Dr.V.B.Narsimaha, "The Performance Comparison of an AODV,DSR, DSDV and OLSR Routing Protocols in Mobile Ad-Hoc Networks", Journal of Computer Applications (JCA) ISSN: 0974-1925, Volume V, Issue 2, 2012

[10].M.UMA，DR.G.PADMAVATHI “A COMPARATIVE STUDY AND PERFORMANCE EVALUATION OF REACTIVE QUALITY OF SERVICE ROUTING PROTOCOLS IN MOBILE ADHOC NETWORKS" journal of Theoretical and Applied Information Technology. Pp. 150-155, 2005- 2009.

[11]. Charles E. Perkins, P Bhagwat , "Highly ynamic
Destination-Sequenced Distance-Vector Routing (DSDV) for mobile computers", SIGCOMM '94 Proceedings of the conference on Communications architectures, protocols and applications, ACM, Pages 234-244 , 1994.

[12].Sapna s. Kaushik \& p.r.deshmukh, Comparison of effectiveness of aodv, dsdv and dsr routing protocols in mobile ad hoc networks", International Journal of Information Technology and Knowledge Management, Vol. 2(2), pp.499-502, 2009.

[13].Prof. Mohit Dua, Prof. Virender Ranga, "Performance Evaluation of AODV, DSDV \& DSR Routing Protocol in Grid Environment", IJCSNS International Journal of Computer Science and Network Security, Foundation of Computer Science USA VOL.9 No.7, pp. 120-126. July 2009

[14].David B. Johnson and David A. Maltz, "Dynamic source routing in adhoc wireless networks," in Mobile computing, T. lmielinski and H. Kmh, Eds, Kluwer Academic, 1996, ch.5.

[15].Staub.T. "Ad-hoc and Hybrid Networks: Performance Comparison of MANET Routing Protocols in d-hoc and Hybrid Networks". Institute of Computer Science and Applied Mathematics, University of Berne, Switzerland, pp.1-38, 2004.

[16].D. Johnson, "Dynamic Source Routing for Mobile Ad Hoc Networks", IEFT MANET Draft, pp. 86-90, April 2003.

[17].Aleksandr Huhtonen , "Comparing ODV and OLSR Routing Protocols", HUT T-110.551 Seminar on Internetworking

[18].Azizol Abdullah, Norlida Ramly, Abdullah Muhammed, Mohd Noor Derahman, "Performance Comparison Study of Routing Protocols for Mobile Grid Environment", IJCSNS International Journal of Computer Science and Network Security, Debashmita Mitra Computer Science and Engineering, CSVTU, India VOL.8 No.2, pp 82-88, February 2008.

[19].Z.Alexander, "Performance Evaluation of AODV Routing Protocol", Proceedings of the third international conference on trends in techologly and engineering, SCC, pp. 178- 183,June 2003,

[20].Thammakit Sriporamanont and Gu liming," Wireless Sensor Network Simulator", Technical report, IDE0602, pp. 200-203 January 2006.

[21].Sukumar Panda, Rahul Mohapatra," Implementation and Comparison of Mobility Models In Ns-2", National Institute of Technology, pp. 160-164Rourkela 2009.

\section{Authors' Profiles}

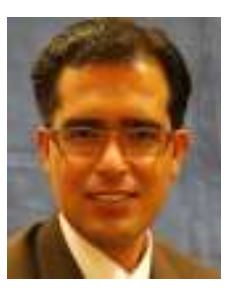

Zafar Mehmood Khattak. He has received his BS degree from Peshawar University, MCS degree from Kohat University in 2006 , MSCS degree from University of Gujrat in 2013. Currently he is working as Lecturer in department of Computer Science/Deputy Manger ORIC in University of Gujrat, Pakistan since 2007. He completed his MSCS in Wireless Networking; his research interest included wireless communication, mobile sensor network and Mobile Ad hoc Networking. He has published 3 papers in International reputed journals.

Dr. Muddesar Iqbal. He earned his $\mathrm{PhD}$ from Kingston University UK in the area of Wireless Mesh Networks in 2009. $\mathrm{He}$ has been serving as Associate Professor in the Faculty of computing and Information technology, University of Gujrat, 


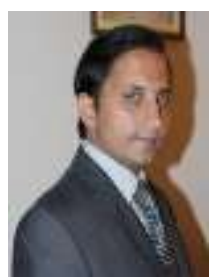

Pakistan since 2010. He won an Award of Appreciation from the Association of Business Executive (ABE) UK for tutoring the prize winner in Computer Fundamentals module. He also received Foreign Expert Certificate from State Administration of Foreign Experts Affairs, People's Republic of China in 2008 against his research collaborations in china. He won another Award of Appreciation from $\mathrm{ABE} U \mathrm{UK}$ for tutoring the prize winner in Information System Project management module in 2010. His research interests span the area of mobile ad hoc routing and security issues, analysis and control of wireless networks, resource management including packet scheduling, and wireless sensor networks and their application like health care and disaster management.

Prof. Xinheng (Henry). He is a professor in Computer networks. Prior to joining UWS, he was working as a senior lecturer at Swansea University. He also worked at Kingston University and Brunel University UK. He has extensive research experience in wireless networking. Over the last few years, he has conducted quite a few research projects funded by EPSRC, TSB (Technology Strategy Board), Welsh Government, China National Natural Science Foundation, China 863, and industry. Some of the developed technologies have been transferred to industry for commercialization. Based on the research project, dozens of papers have been published and over a dozen research students have completed their study. His research interests are includes Wireless mesh networks (WMNs) Wireless sensor networks (WSNs) and Cognitive Wireless Networks.

How to cite this paper: Zafar Mehmood, Muddesar Iqbal, Xingheng Wang,"Comprehensive Experimental Performance Analysis of DSR, AODV and DSDV Routing Protocol for Different Metrics Values with Predefined Constraints", International Journal of Information Technology and Computer Science(IJITCS), vol.6, no.7, pp.24-31, 2014. DOI: 10.5815/ijitcs.2014.07.04 